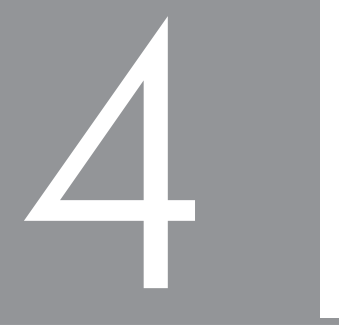

\title{
HARNESSING OPEN EDUCATIONAL RESOURCES TO HELP MANAGE LARGE CLASSES
}

Neil Butcher \& Sarah Hoosen

\section{INTRODUCTION}

Recognition that effective higher education systems play a major role in national economic competitiveness in the increasingly knowledge-driven global economy has placed growing emphasis on the importance of higher education in most countries around the world. As the Organisation for Economic Co-operation and Development (OECD) notes, higher education contributes to social and economic development through four major missions:

- The formation of human capital (primarily through teaching);

- The building of knowledge bases (primarily through research and knowledge development);

- The dissemination and use of knowledge (primarily through interactions with knowledge users); and

- The maintenance of knowledge (intergenerational storage and transmission of knowledge) (OECD 2008:8).

As a result of this trend, higher education is facing immense challenges globally to meet rising enrolment demand. The percentage of the cohort enrolled in higher education has grown from nineteen per cent in 2000 to twenty-six per cent in 2007, which means that there are about 150 million higher education students in the world today (Baty 2009). However, this growth is unevenly distributed, and many countries continue to place strategic emphasis on further rapid growth in higher education enrolments. Typically, though, this growth is not accompanied by equivalent increases in funding or additional personnel to enable universities to accommodate the greater teaching load that such growth generates. A key consequence of this has been a sustained increase in the average size of classes across most 'face-to-face' universities, as they struggle to deal with increasing demand in the face of limited financial resources. These challenges are also often exacerbated in African contexts, which face additional challenges of 
brain drain, underfunding and poor salaries, as well as often underqualified academic staff. The manifold challenges generated by these increases are not described in detail here, as they are covered more comprehensively in other contributions to this volume.

Simultaneously, though, the increasing prevalence of Information and Communication Technologies (ICT) as tools within the education spectrum have "created a universal means of instantaneous contact and simplified scientific communication", and "have helped to concentrate ownership of publishers, databases, and other key resources in the hands of the strongest universities and some multinational companies, located almost exclusively in the developed world" (Altbach, Reisberg \& Rumbley 2009:iv). The past ten years have seen rapid development in ICT and an accompanying explosion of ICT-related activity, as higher education institutions and national systems seek to determine how best to harness ICT to the benefit of students, academics, and countries.

ICT is enabling exponential increases in the transfer of data through increasingly globalised communication systems, and is connecting growing numbers of people through those networks. As a consequence of this growing 'connectedness' and the proliferation of Web 2.0 technologies, there has been an explosion in collective sharing and generation of knowledge. Collective intelligence and mass participation by amateurs in previously specialised disciplinary areas are extending the boundaries of scholarship, while dynamic knowledge creation and social computing tools and processes are becoming more widespread and accepted. This opens the opportunity to create and share a greater diversity of learning resources, thereby accommodating a greater diversity of learner needs. Finally, digitisation of information in all media, combined with increasingly widespread access to this information, has introduced significant challenges in dealing with issues of intellectual property rights (IPR). Copyright regimes, and their associated business models, which worked effectively prior to the explosion of ICT, are rapidly becoming redundant.

Higher education institutions are increasingly viewing investment in ICT - for management and administration, marketing, research, and teaching and learning purposes - as necessary to establish their competitive advantage. This is partly because it is attractive to students (particularly in those parts of the world where young people have increasingly ubiquitous access to ICT), and partly because it is deemed essential by governments, parents, employers, and other key funders of higher education. In many developing countries, however, a paucity of technology, outdated technology, and/or maintenance problems remain challenges, as does access to personal computers for both teaching staff and students. In countries where connectivity and bandwidth are ubiquitous in higher education institutions, this access to the World Wide Web sometimes means that student and teacher are accessing new knowledge or new resources at the same time. Due to expanding telecommunications networks and a rapid decline in the cost and power requirements of hardware, ICT infrastructure and connectivity is however becoming more easily accessible to higher education institutions and students, even in developing countries. 
This rapid ICT development presents both opportunities and challenges for learners who face barriers to traditional means of delivering education, including those enrolled in increasingly large face-to-face classes. Digital systems are highly adaptable and flexible, and can be personalised to a wide variety of learning needs. Within this context, there is a significant potential role for effective use of ICT and the access it provides to Open Educational Resources (OER) and other openly licensed resources in helping to ameliorate the effects of growing student cohorts by rethinking the way in which teaching and learning is organised in environments where, increasingly, students have ubiquitous access to ICT devices and the connectivity necessary to access the Internet. However, before discussing how ICT might be used to tackle the effect of growing class sizes, it is worth exploring the potential educational value of OER.

\section{A RATIONALE FOR ENGAGING WITH OER}

At its core, OER is a simple legal concept: it describes educational resources that are openly available for use by educators and students, without an accompanying obligation to pay royalties or licence fees. Different options are emerging that can be used to define how OER are licensed for use (and adaptation as appropriate), some of which simply allow copying and others that make provision for users to adapt the resources that they use. The best known of these are the Creative Commons licences, which provide legal mechanisms to ensure that people can retain acknowledgement for their work while allowing it to be shared, and can choose to restrict commercial activity if they so wish or, if appropriate, prevent their work from being adapted (although this may be difficult to enforce legally).

The emergence of open licences has occurred in an effort to protect an author's rights in environments where content (particularly when digitised) can so easily be copied and shared on the Internet without permission. Digitisation of information in all media, combined with increasingly widespread access to this information, has introduced significant challenges regarding how to deal with issues of intellectual property such as copyright. The ability for anyone to copy and share content once it has been digitised creates both opportunities and challenges for higher education providers. The main challenges relate to the ease with which digitised content can be copied and shared, with or without the permission of the copyright holder. Thus, open licences seek to ensure that this copying and sharing happens within a structured legal framework that is more flexible than the automatic 'all-rights reserved' status of copyright.

Many proponents of OER consider that a key benefit of open content is that it is 'free' (that is, bandwidth costs aside, it does not cost anything to download). This is literally true for the end-user: by definition, open content may be shared with others without asking permission and without paying licence fees. Yet, there are some important cost considerations to be taken into account.

Effective harnessing of OER first requires that institutions invest systematically in programme, course, and materials development/acquisition. Costs will include wages 
for time spent in developing curricula and materials, adapting existing $O E R$, dealing with copyright licensing (if material is not openly licensed), and so on. There are also associated costs, such as ICT infrastructure (for authoring and content-sharing purposes), bandwidth expenses, running workshops and meetings when content development teams meet, and so on.

All educational institutions should be making these investments on an ongoing basis to improve the quality of teaching and learning. Incorporating effective adaptation and use of OER is the most cost-effective way to invest in materials design and development, because it eliminates unnecessary duplication of effort by building on what already exists elsewhere; takes advantage of pooled alternative resources to meet accessibility obligations; removes the costs of copyright negotiation and clearance; and, over time, can engage open 'communities of practice' in ongoing quality assurance and improvement.

Despite the great potential of OER, there are several challenges with regard to using and creating OER in an African context. For example, being able to access OER requires adequate ICT infrastructure and a robust and fast connection to the Internet, which is still lacking in many African institutions. Available OER may also not always match methods or subject matter as taught locally, so there may be a need to train and capacitate staff to source and adapt OER. In addition, due to unfamiliarity with OER implementation models, there is a need for lobbying about the benefits of OER in order to encourage buy-in from African academics to use them.

Nevertheless, given the challenge of growing enrolment with limited increases in resources, combined with the ongoing rollout of ICT infrastructure in higher education institutions, it is becoming increasingly important for educational institutions to support, in a planned and deliberate manner, the development and improvement of curricula; ongoing programme and course design; planning of effective contact sessions with students; meeting the needs of a greater diversity of learners; development of quality teaching and learning materials; and design of effective assessment. All of these activities are aimed at improving the teaching and learning environment, while managing cost through increased use of resource-based learning. OER manages this investment and the resulting copyright issues in a way that creates significant opportunities to support ongoing improvements in the teaching and learning process. It also helps significantly in meeting the challenge of managing growing class sizes.

\section{LINKING OER TO RESOURCE-BASED LEARNING}

Significant emphasis has been placed on the quality of OER in OER discussions. This renders the concept of resource-based learning of particular interest. Yet, until recently, debates about OER have typically made little reference to resource-based learning. This may be because the emphasis in most international OER discussion has been on the sharing and licensing of existing materials, a significant proportion of which has 
included simply sharing lecture notes and PowerPoint presentations used in face-toface lectures.

What does the concept of resource-based learning mean, in essence? It means moving away from the traditional notion of using the 'talking teacher' to communicate curriculum - a varying but significant proportion of communication between students and educators does not occur face to face, but happens instead through the use of different media (such as text, videos, simulations and animations), as appropriate. Importantly, the face-to-face contact that does take place typically does not involve simple transmission of information from educator to student; instead, it involves various forms of student support, for example, academic debate, tutorials, peer group discussion, or practical work.

Resource-based learning is not a synonym for distance education. Rather, resourcebased learning provides a basis for transforming teaching culture across all educational systems, in order to enable those systems to offer better quality education to significantly larger numbers of students. Many courses and programmes at all levels of education now incorporate extensive use of instructionally designed resources, as educators have recognised the limitations of relying exclusively on lecture-based strategies for communicating information to students. Given the centrality of lecturing as a communication mode at most face-to-face universities, it is important to unpack this issue in more detail.

\section{RETHINKING THE ROLE OF LECTURING IN LARGE CLASSES}

At many face-to-face universities, even those with increasingly large classes, the lecture method remains the most widely used form of presenting information to students. Using this method, the lecturer is most often (although not always) established as the expert with a body of knowledge to communicate to students. Typically, students remain predominantly silent participants, with their activity being largely limited to taking notes. The prevalence of lectures often has less to do with learning and more with a long tradition of viewing it as a principal form of teaching, coupled with the pressures created by increasing enrolments; the efficiency (time and cost) of lectures; the familiarity of the format to lecturers (a function of lecturers' preferred teaching styles rather than students' preferred learning styles); and the time-efficiency of preparing lectures.

However, many academics believe that the lecture method of teaching may be ineffective when compared to active learning methods, particularly when it is employed as the dominant mode of information transmission. In a 2006 review of forty papers by ninety-nine authors on active versus passive learning, Siriopoulos and Pomonis (2006) noted that ten per cent preferred lectures, while eighty-eight per cent favoured alternative teaching methods, and two per cent combinations of the two. Methods promoting active learning by students are based on the constructivist view that, for 
meaningful learning to occur, students must actively engage with subject matter through activities such as discussion, problem solving and hands-on activities.

Research about the learning process indicates that more effective learning occurs when students are actively engaged; have opportunities for interaction with others; are presented with challenging situations or questions that require critical thinking skills; and are surrounded by a nurturing learning environment (Ray 2004). Proponents of active learning methods note that a main weakness of the lecture method is that it allows students to be passive recipients of information, because there is no mechanism to ensure that they are intellectually engaged with the material (Hansen \& Stephens 2000). Although there are, of course, approaches to lecturing that can generate greater levels of engagement with students - for example, creatively stimulating discussion or using academic texts to create active learning environments - these approaches are also increasingly difficult to implement effectively as class sizes expand. In the typical large class, the instructor lectures to usually anonymous students who take notes without the opportunity to ask questions or interact with the instructor or fellow students (Lass, Morzuch \& Rogers 2007). In this way, students become dependent on the lecturer to tell them what they need to know.

Furthermore, lectures predominantly emphasise learning by listening, which puts students who have other learning styles at a disadvantage. Undoubtedly, lectures will suit some students, but it would be erroneous to assume that all (or even most) students are motivated intrinsically by the material or the inspirational style of the lecturer (Learningspark nd). Data from first-year undergraduate economics students in the 1998/99 academic year at the University of Ancona in Italy, for example, provides evidence that, once self-study time is controlled for, the positive and significant effect of lecture attendance disappears for some courses (Bratti \& Staffolani 2002).

The need for active student participation in the learning process demands the development and use of more complex teaching methods, such as problem-based learning, field studies, cooperative, collaborative, and group learning, interactive computer simulations, and other innovative teaching modes. For example, McKenzie and Swords (2000, in Siriopoulos \& Pomonis 2006) demonstrate that the skills and knowledge requirements implied by the modern business environment are unlikely to be met by traditional, lecture-driven business education programmes. They suggest technology integration in teaching to develop greater learner autonomy, without compromising content and in ways that enhance interaction and enrich teacherlearner and learner-learner relationships. Cherry (2004) proposes the use of reading guides as an alternate method of delivering content, so as to allow more time for active learning.

In addition, as a teaching method, the lecture does not always bring about maximum attainment of certain types of learning outcomes. Lecturing may not be suited to teaching higher orders of thinking such as application, analysis, synthesis, or evaluation, or for influencing attitudes or values - all of which are typically regarded as key attributes 
of effective higher education graduates. Lectures are also not well suited to teaching complex, abstract material. Speech skills, motor skills and cooperative group thinking, for example, are difficult to teach with the lecture method. Students can develop such skills properly only through practice. Moreover, the formal lecture alone is generally not appropriate for presenting material above the comprehension level of the cognitive domain. As it allows for little or no verbal participation by students, the formal lecture may also be inefficient for comprehension-level lessons where concepts and principles are developed (Learningspark nd).

This is not to say that there is no role for lectures, or that the lecture is not a good teaching technique. For some content, particularly if a primary objective is to disseminate information, introduce material not readily available elsewhere, or provide inspiration, the lecture method is appropriate and potentially effective. It is particularly suitable for introducing a subject: a brief introductory lecture can give direction and purpose to a demonstration; prepare students for a discussion or debate; and serve to arouse interest in a subject. An effective lecture may also stimulate discussion or provide a basis for additional study. Lectures can also be useful as a way to supplement material from other sources or to provide information that is difficult to obtain in other ways, particularly if students do not have the time required for research or if they do not have access to reference material. In subject areas where information is available in widely scattered places (textbooks, journals, tapes), the lecture allows the instructor to summarise and emphasise pertinent material (Learningspark nd). Cooper (1982) stresses that lectures alone should rarely be used without some opportunity for student participation. He notes that lectures may be used to provide certain kinds of information, perhaps reinforcing or explaining written content, but not to "reiterate materials learners can read" (ibid:39).

In the context of lecturing to large classes, several authors provide suggestions that lecturers can adopt to reduce the negative features of large lectures. Most commonly, there is a call for multiple teaching strategies in order to accommodate the different learning styles of students. Thus academics are increasingly required to do more than just write up lecture notes and walk into class. They should first be aware of the limitations of large classes, and then plan their teaching and learning activities to compensate for those limitations (Arbour, Karras \& Lee 2010). This is particularly important in developing world contexts, where a lack of equipment and teaching materials, rising student enrolments, a shortage of academics, and poor infrastructure may result in an over-reliance on the lecture method. Improved instructional technology - and particularly the development of the Internet and the World Wide Web - has provided opportunities for academics to use these technologies to improve teaching and learning. The proliferation of available resources makes it possible to spread the communication of content across a wider range of methods, offering students multiple modes of learning, rather than being limited to lectures. Given the proliferation of OER and ICT infrastructure on university campuses, these methods are not only increasingly affordable to implement and accessible to students; they also provide many alternatives 
for designing creative teaching and learning environments that can offset the problems associated with growing student enrolments.

Effective teaching and learning constitute the ultimate goal of academic instructors. The particular method adopted will depend on many factors, including the age and developmental level of students; what they already know and what they need to know to succeed with the lesson; the subject-matter content; the objective of the lesson; the available human, time, space, and material resources; and the physical setting (Kizlik 2011 ). As Becker and Watts (1996:52, quoted in Siriopoulos \& Pomonis 2006) note, "great orators should continue to lecture, but [...] the rest of us should consider using a wider variety of teaching methods [...] to actively engage students." Lectures are simply one method of instruction, useful for particular types of engagement.

\section{WHAT IMPLICATIONS DOES THIS HAVE FOR UNIVERSITIES?}

Most universities describe their mandate as threefold: research, teaching and learning, and outreach. Whereas research and collaborative partnerships often enjoy priority on an institution's agenda, teaching and learning may at times receive less institutional focus, because it is assumed that it is strictly the domain of qualified academic staff. However, in most cases, academics are appointed on the basis of their disciplinary and research expertise, rather than a proven ability to teach effectively, and are likely to need support when it comes to adopting new ways of teaching.

As noted, one of the core functions of a university is effective teaching and learning, which requires appropriate investment in curriculum and course design and materials development, as well as ongoing evaluation and regular renewal. At the same time, teaching staff often have to deal with ever-increasing class sizes and a growing diversity of learners. Institutions therefore need to make better use of the resources they have. Given this reality, and for the reasons argued above, the primary role of academic teaching staff should not necessarily be delivery of content in the form of lectures: this can be managed more effectively by the development and provision of learning resources. Well-designed learning resources typically require much greater individual engagement by students with information, ideas, and content than is possible in a large-scale contact lecture. Teaching staff can harness OER to facilitate more effective teaching and learning in ways that save time and that enable students to take greater control of their own learning - engaging more with core resources in their own time and at their own pace. Open licences that encourage activity and creativity on the part of students through the reuse and adaptation of content can make a significant contribution towards creating more effective learning environments. This freedom to modify also provides an unprecedented opportunity to adapt curricula so as to engage a far greater diversity of learners, who would otherwise face barriers to learning due to large class sizes, language, cultural conventions, or disabilities. Freed from being the primary deliverers of content, teaching staff can then use their time more strategically to nurture meaningful engagement and debate, and to reflect upon their own curriculum 
and pedagogic assumptions and practice with a view to critical reflexive practice. Face-to-face time with students can then be better used to support engagement and to nurture discussion, debate and practical application, or to support student research activities, thereby providing students with tools to advance their own understanding.

In developing curricula and learning resources, academics have always engaged with what is already available, often prescribing existing textbooks; building on previous iterations of a course taught by predecessors or colleagues; and creating reading lists of published articles. Even at distance education institutions, which have a long history of materials development, it is arguably a rare occurrence to develop completely new materials with no reference to what already exists. Because OER removes restrictions around copying and adapting resources, it can reduce the cost of accessing educational materials. These materials can then be used to supplement and enrich courses, which is particularly useful when there are large course cohorts. OER also removes the need for royalty payments for text books and other educational materials, as well as the time-consuming processes of procuring permission to use copyrighted material. Even where teaching staff produce new materials, their ability to draw inspiration and ideas from other people's openly accessible teaching materials can serve to increase quality without adding cost. Perhaps more importantly, it opens greater possibilities for adapting existing resources to be more relevant to local contextual and cultural needs while also bearing in mind the accessibility needs of learners. OER has potential to build capacity by providing institutions and teaching staff access, at relatively low cost, to the means to create and adapt high-quality teaching and learning materials. This can be harnessed to develop competence in producing such materials and carrying out the necessary instructional design to integrate them into high-quality programmes of learning. Increasingly, while teaching staff are expected to have the knowledge and skills to teach a broad spectrum of subjects, they often lack the time to revisit and modify curriculum and educational materials on a regular and systematic basis. The transparency provided by OER (resources produced by staff are shared openly) places social pressure on institutions and teaching staff to increase quality; allows them to better coordinate curricula; and provides resources for students' learning and for academic planning.

Openly licensed educational materials therefore have tremendous potential to contribute to improving the quality, accessibility, and effectiveness of education, while at the same time serving to restore a core function of education: sharing knowledge. All of these benefits can be harnessed to mitigate the negative educational effects of large class sizes.

On the teaching and learning side, universities that succeed in managing large classes most effectively are likely to do so mainly by focusing not on content itself (which is increasingly available in large volumes online), but rather on developing their institutional capacity to guide students successfully through educational resources via well-designed teaching and learning pathways; offer effective support to students 
(whether that be in practical sessions, tutorials, individual counselling sessions, or online); and provide intelligent assessment and critical feedback to students on their performance (ultimately leading to some form of accreditation). Historically though, academics have often been actively encouraged to protect their intellectual capital. Their incentives to publish are clear, but sharing teaching practices, approaches or materials is not necessarily a common practice. It is thus important to find ways to shift this culture and to encourage ways of sharing materials that are not threatening.

In addition, incentive structures often reward individual, rather than collaborative, activity and encourage production of 'new' materials. While there are sometimes good reasons for a faculty member to develop materials from scratch, such processes may often duplicate ongoing work taking place in global knowledge networks that are engaged in facilitating increasingly creative forms of collaboration and information sharing. Consequently, it is opportune for universities to think strategically about the extent to which their policies, practices, and institutional culture reward individual endeavour over collaboration and create inefficiencies by prizing, in principle, creation of 'new' materials above adaptation and use of existing materials and content. As the amount of content freely accessible online proliferates, such approaches to procuring materials are unnecessarily wasteful. Thus, there may be merit in ensuring that incentive structures and quality assurance processes include selection and use of existing content as well as development of new content.

\section{CONCLUSION}

The challenge of increased student enrolments and reduced budgets in higher education opens an opportunity to harness OER to help with the management of large classes. Perhaps the greatest challenge is how to actively engage students in learning, as teaching has to become "value-added intelligence producing" if universities are to survive as knowledge-producing organisations (CHE 2001). Adopting a resourcebased approach using OER can assist in overcoming some of these challenges by channelling energy towards multiple modes of instruction, rather than limiting it to a traditional lecture format. OER helps create a diversity of learning resources, accommodating a greater diversity of learner needs. In addition, it facilitates better use of resources by providing students with the opportunity to engage with material out of class, and instructors to use contact time to facilitate knowledge acquisition and understanding. Adopting such an approach requires a shift in mindset: creating transparency and an open culture of sharing, and acknowledging that using only traditional lecturing as a means of communicating information is ineffective and not optimal for learning in large-class contexts.

With this in mind, universities adopting such an approach will likely need to review and adjust their existing policies and staff incentive schemes to ensure that they encourage teaching staff to invest time in ongoing curriculum design; the creation of effective teaching and learning environments within courses and programmes; 
and development of high-quality teaching and learning materials. Key policies include those that govern staff incentives, rewards, and promotion. Also important for consideration is the process of determining staff workload, and how it affects the allocation of time to different kinds of tasks. In addition, it will be useful to make provision in internal quality assurance processes for assessing and reviewing the role and use of educational resources in improving the quality of teaching and learning, as well as how these are integrated into curricula to form meaningfully coherent learning pathways. This should include establishing and maintaining a rigorous internal process for validating the quality of educational materials prior to their publication as OER. Using such approaches, universities would be in a much better position to deal with the effects of growing student enrolments, and would manage this growth in ways that are both educationally effective and financially sustainable. 